\title{
Integration of a III-V light emitter on a silicon photonic IC through transfer printing
}

\author{
A. De Groote ${ }^{1,2, *}$, P. Cardile ${ }^{3}$, A.Z. Subramanian ${ }^{1,2}$, A.M. Fecioru ${ }^{4}$, \\ C. Bower ${ }^{5}$, D. Delbeke ${ }^{1,2}$, R. Baets ${ }^{1,2}$, G. Roelkens ${ }^{1,2}$ \\ ${ }^{1}$ Photonics Research Group, Department of Information Technology, Ghent University - imec, Ghent, Belgium \\ ${ }^{2}$ Center for Nano- and Biophotonics (NB-Photonics), Ghent University, Ghent, Belgium \\ ${ }^{3}$ Center for Microsystems Technology, Ghent University - IMEC, Ghent, Belgium \\ ${ }^{4} \mathrm{X}$-Celeprint Limited, Cork, Ireland \\ ${ }^{5} \mathrm{X}$-Celeprint Inc., Research Triangle Park, NC, USA \\ *Email: andreasdegroote@intec.ugent.be
}

\begin{abstract}
For the first time, III-V opto-electronic components are coupled to silicon waveguide circuits using transfer-printing. Efficient III-V material usage is enabled in silicon photonics by providing III-V only where needed. We present transferprinted single-spatial-mode LEDs coupling to silicon-on-insulator waveguides as first examples of this technology.
\end{abstract}

\section{INTRODUCTION}

Silicon photonics revolves around using CMOS compatible fabrication techniques in order to profit maximally from the decades of investment done by integrated electronics. Today, high quality passive components and active components such as modulators and detectors are available. Due to the indirect band gap of silicon, the integration of efficient sources in silicon photonic ICs is still a problem to tackle. While today individual III-V lasers are being assembled onto silicon photonic ICs, this is not a scalable approach when arrays of sources need to be integrated. Therefore the heterogeneous integration of III$\mathrm{V}$ semiconductors through a bonding technique has been the subject of much research, in which case the alignment of the III-V component with respect to the silicon photonic IC is lithographically determined on wafer scale. Classical heterogeneous integration approaches rely on die-to-wafer bonding using millimeter-sized optical dies. In many cases the fraction of the chip real estate occupied by the III-V is much smaller than this die size. Therefore such an approach does not allow for efficient use of III-V material. The dense integration of different III-V layer stacks is also problematic for the same reason [1]. In 2004, Menard et al. [2] proposed a novel technique, transfer printing, where thin film components or layer stacks (hereafter referred to as a coupon) were transferred from a source substrate to a target substrate. With this technique several components were successfully printed to a blank silicon/glass target [3]-[5]. In order to be able to pick the coupon with a soft elastomeric stamp, it has to be undercut such that it is only supported by tethers. As described in [6] the adhesion of the elastomeric stamp depends on the velocity of the stamp. Hence, pick-up is done by moving up the stamp rapidly while releasing the stamp slowly prints the coupon. While there are many similarities with a pick-and-place technique, the main advantage of transfer printing is that coupons can be printed in a massively parallel way, using a patterned stamp. Next to the high-throughput, transfer printing also allows for very efficient use of source material, since one only provides small III-V coupons (tens to hundreds of microns in size) where they are needed. One can also densely co-integrate multiple III-V material stacks. In this paper, we present the first InPbased opto-electronic components transfer printed on and coupled to a silicon-on-insulator waveguide circuit.

\section{TRANSFER PRINTING PROCESS FLOW}

The transfer printing process flow for InP-based membrane devices is shown in Figure 1. The InP layer stack consists of a release
InGaAs layer $(1 \mu \mathrm{m})$ with the device layers on top (InGaAsP multiquantum-wells with $60 \mathrm{~nm}$ InP cladding). A sacrificial InGaAs / InP $(100 \mathrm{~nm} / 1 \mu \mathrm{m})$ layer pair is added for mechanical robustness. The $40 \mu \mathrm{m}$ wide coupons are etched into a double mesa. The structure is etched down to the substrate in the locations where the tethers will anchor. Afterwards, the coupon is encapsulated in photoresist and anchored to the InP substrate as shown in Figure 1d and the InGaAs release layer is etched from the side using an $\mathrm{FeCl}_{3}: \mathrm{H}_{2} \mathrm{O}$ solution. After an etch of 2 hours, the $40 \mu \mathrm{m}$ wide coupons were undercut completely with the thin InP cladding layer intact. Now the coupons

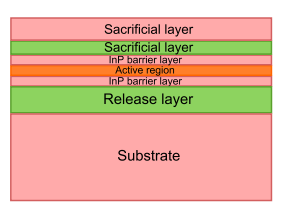

(a) InP starting layer stack

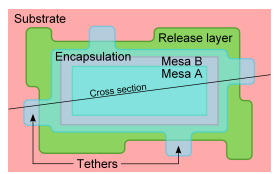

(d) Top view after encapsulation and tether definition

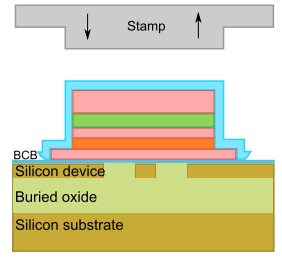

(g) Printing of the III-V coupon to the SOI target substrate

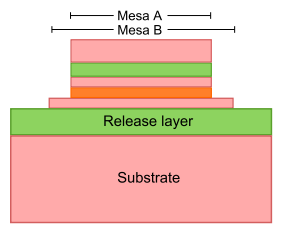

(b) Coupon patterning

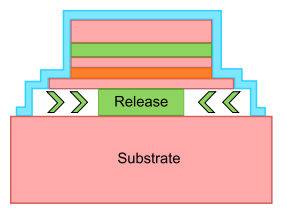

(e) Release etch

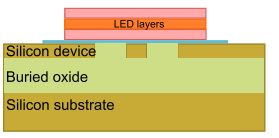

(h) Removal of encapsulation and of sacrificial layers

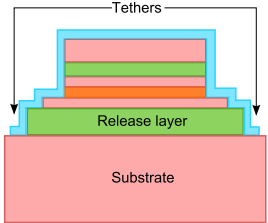

(c) Encapsulation and tether definition

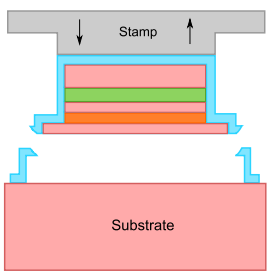

(f) Pick-up of the III$\mathrm{V}$ coupon from the InP source substrate

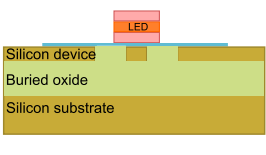

(i) Definition of LED
Fig. 1: Transfer printing process flow 


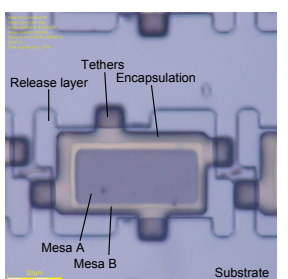

(a) Coupons on source wafer

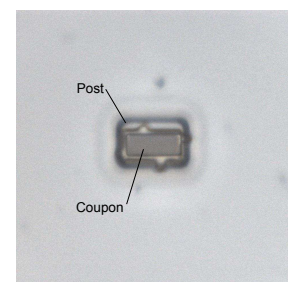

(b) Picked-up coupon on stamp

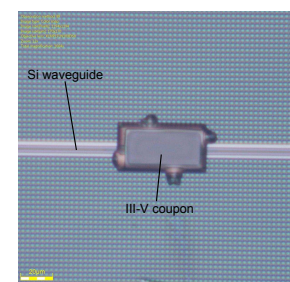

(c) Printed coupon on target wafer
Fig. 2: Microscope images of the coupons before, during and after transfer printing

can be transferred using the elastomeric stamp, as indicated in the microscope images of figure 2 . The target samples are planarized SOI chips with $220 \mathrm{~nm}$ thick waveguide circuits with $100 \mathrm{~nm}$ top $\mathrm{SiO}_{2}$. A cross section of a transfer printed coupon onto an SOI waveguide circuit is shown in Figure 3a. The bond relies purely on Van der Waals forces. Because the CMP planarization leave steps of $\sim 20 \mathrm{~nm}$ on the surface, voids show up at the interface. This issue can be resolved by using a $50 \mathrm{~nm}$ thick DVS-BCB adhesive bonding layer. This 50 $\mathrm{nm}$ thick layer planarizes the target sample and provides a strong bond, as can be seen in figure $3 \mathrm{~b}$. After transfer printing the coupon to a DVS-BCB coated SOI waveguide circuit at room temperature, the photoresist encapsulation is removed with an oxygen plasma. The bonding layer is fully cured at $250^{\circ} \mathrm{C}$ for 1 hour and the sacrificial InP-InGaAs layer pair is removed in $\mathrm{HCl}$-based and $\mathrm{H}_{2} \mathrm{SO}_{4}$-based etchant, without delamination. The Van der Waals bonded samples did delaminate, highlighting the benefit of using a thin bonding agent. Afterwards the membrane is patterned. This ensures the devices are lithographically aligned to the SOI and do not rely on the $1.5 \mu \mathrm{m} 3 \sigma$ misalignment of the transfer printer. [7].

\section{TRANSFER PRINTED LIGHT EMITTING DEVICE}

As a proof-of-principle for the transfer printing of III-V photonic components to SOI waveguide circuit, we present a single-spatialmode broadband light emitting device, shown in figure 4. Such a component is of great interest for on-chip spectroscopic sensing applications, because of its low power consumption, simplicity and robustness compared to tunable lasers and superluminescent LEDs, as discussed in [8]. A $1310 \mathrm{~nm}$ pump laser pumps the LED through the silicon waveguide. Through an adiabatic taper, the pump light is coupled to the III-V membrane, where it is strongly absorbed. Of the

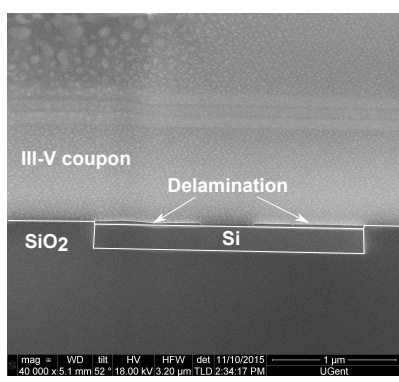

(a) Transfer printed coupon on planarized SOI using Van der Waals bonding

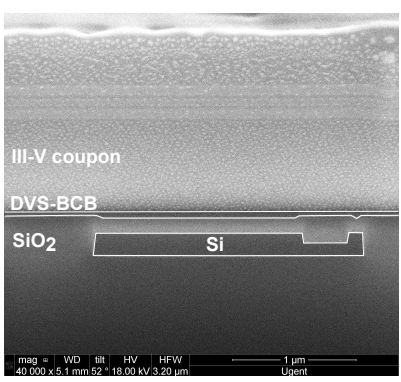

(b) Transfer printed coupon on planarized SOI using a DVS$\mathrm{BCB}$ adhesive bonding agent
Fig. 3: Cross sections of transfer printed coupons on planarized SOI waveguide circuits

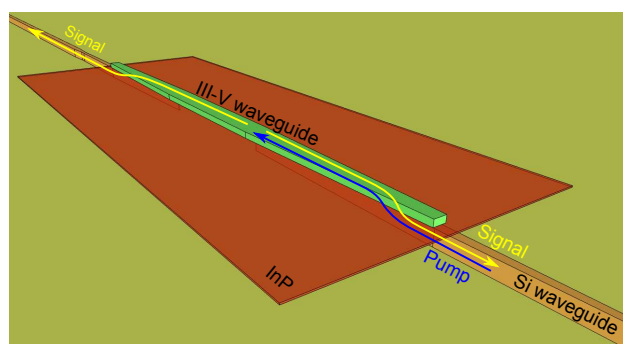

Fig. 4: Illustration of the optically pumped LED.

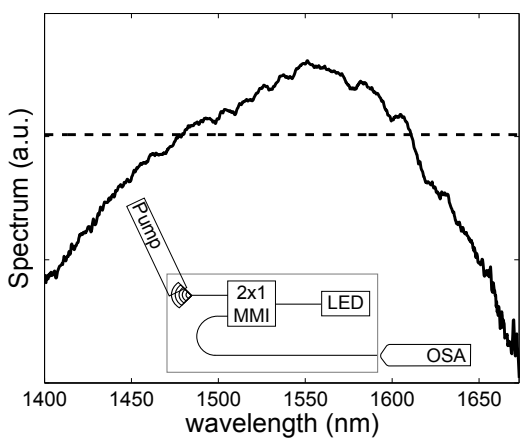

Fig. 5: Measured spectrum of transfer printed, optically pumped LED.

resulting spontaneous emission (around $1550 \mathrm{~nm}$ ), a large fraction ( $\sim 10 \%$ for the TE polarized light in this membrane, as simulated by FDTD simulations) couples to the co-propagating and counterpropagating waveguide modes. This fundamental mode then couples down to the silicon waveguide through the adiabatic couplers. The spectrum of the spontaneous emission collected in reflection is shown in Figure 5, with the measurement setup shown in the inset. While an exact measurement of the efficiency was not possible on our chips, we are convinced it is of the same order as previously reported by traditional bonding methods. The $3 \mathrm{~dB}$ bandwidth of $130 \mathrm{~nm}$ is large and comparable as well to the values reported in [8].

\section{Summary}

For the first time, transfer printing is used to integrate a III$\mathrm{V}$ opto-electronic component which couples to a silicon photonic integrated circuit. Transfer printing allows for the efficient use of III-V material and high-throughput integration. We discussed the transfer printing process flow, using aqueous $\mathrm{FeCl}_{3}$ to etch the InGaAs release layer. With the introduction of a $50 \mathrm{~nm}$ thick DVS-BCB bonding layer, a strong bond was achieved. Successful demonstration of a transfer printed light emitting device is reported. This work is a stepping stone towards the cost-effective integration of III-V opto-electronic components onto complex silicon photonic integrated circuits.

\section{REFERENCES}

[1] A. De Groote et al., Opt. Lett. 39, 4784-4787 (2014)

[2] E. Menard et al., Appl. Phys. Lett. 84, 5398-5400 (2004)

[3] H. Yang et al., Nature Photon. 6, 615-620 (2012)

[4] J. Justice et al., Nature Photon. 6, 610-614 (2012)

[5] A. Trindade et al., Appl. Phys. Lett. 103, 253302 (2013)

[6] M. Meitl et al., Nat. Mater. 5(1), 33-38 (2006)

[7] C. Bower et al., Proc. IDW, 1203-1206 (2010)

[8] A. De Groote et al., Proc. GFP, 31-32 (2015) 